\title{
Direct Electrophilic Acylation of N-Substituted Pyrazoles by Anhy- drides of Carboxylic Acids
}

Ilya V. Taydakov*

Sergey S. Krasnoselskiy

Synthesis 2013, 45, 2188.

In Scheme 4, an oxygen atom in the structure of the anhydride is missing. The correct Scheme is shown below.

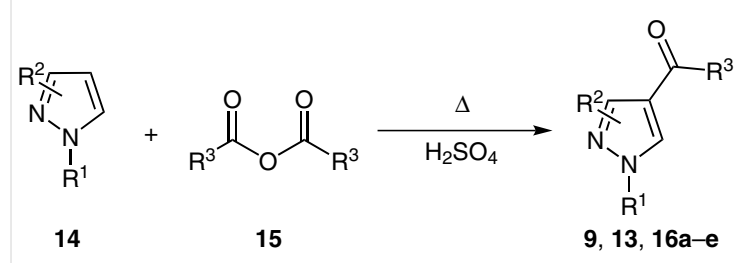

The authors apologize. 